\title{
The Cayley transform of the generator of a polynomially stable $C_{0}$ - semigroup
}

\author{
MASASHI WAKAIKIID
}

Abstract. In this paper, we study the decay rate of the Cayley transform of the generator of a polynomially stable $C_{0}$-semigroup. To estimate the decay rate of the Cayley transform, we develop an integral condition on resolvents for polynomial stability. Using this integral condition, we relate polynomial stability to Lyapunov equations. We also study robustness of polynomial stability for a certain class of structured perturbations.

\section{Introduction}

Consider a $C_{0}$-semigroup $(T(t))_{t \geq 0}$ on a Hilbert space with generator $A$, and suppose that $(T(t))_{t \geq 0}$ is polynomially stable with parameter $\alpha>0$, that is, $(T(t))_{t \geq 0}$ is uniformly bounded, the spectrum of $A$ is contained in the open left half plane, and there exists $M>0$ such that for all $t>0$,

$$
\left\|T(t) A^{-1}\right\| \leq \frac{M}{t^{1 / \alpha}} .
$$

We consider the following question: Is polynomial decay of $(T(t))_{t \geq 0}$ passed to the Cayley transform $A_{d}:=(I+A)(I-A)^{-1}$ ? The quantitative behavior of the operator norm $\left\|T(t) A^{-1}\right\|$ has been extensively studied; see, e.g., [2-4,6,20,28]. A discrete analogue, the quantified Katznelson-Tzafriri theorem, has also been investigated in $[7,22,29,30]$. However, to the author's knowledge, it has not been well established whether and how polynomial decay of $(T(t))_{t \geq 0}$ in the continuous setting yields the decay of the corresponding Cayley transform in the discrete setting. The purpose of this paper is to show that polynomial decay of a $C_{0}$-semigroup is preserved under the Cayley transformation in a certain sense.

Applications of the Cayley transform of a semigroup generator arise in numerical analysis [26] and system theory [32, Section 12.3]. In the finite-dimensional case, a matrix and its Cayley transform share the same stability properties, but this does not hold in the infinite-dimensional case. In fact, in the Banach space setting, the Cayley transform of the generator of even an exponentially stable $C_{0}$-semigroup may not be power bounded [15, Lemma 2.1]. For the case of Hilbert spaces, it is still unknown 
whether the corresponding Cayley transform is power bounded for every generator of a uniformly bounded $C_{0}$-semigroup, as mentioned in Section 5.5 of [5]. However, some sufficient conditions for Cayley transforms to be power bounded have been obtained; see, e.g., $[12,14,15,17]$. In particular, it is well known that $A$ generates a $C_{0}$-semigroup of contractions on a Hilbert space if and only if the corresponding Cayley transform is a contraction [21, Theorem III.8.1]. We refer the reader to the survey [13] for more details.

In this paper, we prove that if $(T(t))_{t \geq 0}$ is a polynomially stable $C_{0}$-semigroup with parameter $\alpha>0$ on a Hilbert space with generator $A$ such that its Cayley transform $A_{d}$ is power bounded, then there exists $M_{d}>0$ such that for all $n \in \mathbb{N}$,

$$
\left\|A_{d}^{n} A^{-1}\right\| \leq M_{d}\left(\frac{\log n}{n}\right)^{\frac{1}{\alpha+2}} .
$$

We also show that in some cases, such as when $A$ is normal, the logarithmic correction can be omitted. Moreover, we give a simple example of a normal operator $A$ for which the decay rate $1 / n^{1 /(\alpha+2)}$ cannot be improved.

To obtain the decay estimate of Cayley transforms, we extend the Lyapunov-based approach developed by Guo and Zwart [17]. In [17], uniform boundedness and strong stability of a $C_{0}$-semigroup have been characterized in terms of the solution of a certain Lyapunov equation. The integral conditions on resolvents obtained in $[16,31]$ for uniform boundedness and in [33] for strong stability play an important role in this Lyapunov-based approach. Therefore, we first obtain a similar integral condition for polynomial stability. By means of this integral condition, we next relate polynomial stability to the Lyapunov equation used in [17]. Finally, we estimate the decay rate of the Cayley transform, by using the solution of the Lyapunov equation.

As another application of the Lyapunov-based approach, we consider the following robustness analysis of polynomial stability: If $A$ generates a polynomially stable semigroup, then does $A+r A^{-1}$ also generate a polynomially stable semigroup for every $r>0$ ? Robustness of polynomial stability has been studied in [23-25,27]. In these previous studies, perturbations are not structured, but the norms of the perturbations are assumed to be bounded in a certain sense. In contrast, the class of perturbations we consider is limited to $\left\{r A^{-1}: r>0\right\}$, but we do not place any norm conditions for perturbations. We show that if $A$ generates a polynomially stable semigroup with parameter $\alpha>0$, then for every $r>0, A+r A^{-1}$ also generates a polynomially stable semigroup with the same parameter $\alpha$ in the case $\alpha>2$ and with parameter $\alpha+\varepsilon$ for arbitrary small $\varepsilon>0$ in the case $\alpha<2$. If $\alpha=2$, then a logarithmic factor appears in the rate of decay.

This paper is organized as follows. In Sect. 2, we collect some preliminary results on polynomial stability. In Sect. 3, we present an integral condition on resolvents for polynomial stability and then connect this stability to Lyapunov equations. In Sect. 4, we study the decay rate of the Cayley transform of the generator of a polynomially stable $C_{0}$-semigroup. Section 5 contains the robustness analysis of polynomial stability for the class of perturbations $\left\{r A^{-1}: r>0\right\}$. 
Notation Let $\mathbb{C}_{-}:=\{\lambda \in \mathbb{C}: \operatorname{Re} \lambda<0\}$ and $i \mathbb{R}:=\{i \eta: \eta \in \mathbb{R}\}$. The closure of a subset $\Omega$ of $\mathbb{C}$ is denoted by $\bar{\Omega}$. For real-valued functions $f, g$ on $J \subset \mathbb{R}$, we write $f(t)=O(g(t))$ as $t \rightarrow \infty$ if there exist $M>0$ and $t_{0} \in J$ such that $f(t) \leq M g(t)$ for every $t \geq t_{0}$, and similarly, $f(t)=o(g(t))$ as $t \rightarrow \infty$ if for every $\varepsilon>0$, there exists $t_{0} \in J$ such that $f(t) \leq \varepsilon g(t)$ for every $t \geq t_{0}$. Let $X$ be a Banach space. For a linear operator $A$ on $X$, we denote by $D(A)$ and $\operatorname{ran}(A)$ the domain and the range of $A$, respectively. The space of bounded linear operators on $X$ is denoted by $\mathcal{L}(X)$. For a closed operator $A: D(A) \subset X \rightarrow X$, we denote by $\sigma(A)$ and $\varrho(A)$ the spectrum and the resolvent set of $A$, respectively. Let $\tilde{\sigma}(A)$ be the extended spectrum of $A$ defined by

$$
\tilde{\sigma}(A):= \begin{cases}\sigma(A) & \text { if } A \in \mathcal{L}(X) \\ \sigma(A) \cup\{\infty\} & \text { if } A \notin \mathcal{L}(X) .\end{cases}
$$

For $\lambda \in \varrho(A)$, the resolvent operator is given by $R(\lambda, A):=(\lambda-A)^{-1}$. Let $H$ be a Hilbert space. The inner product of $H$ is denoted by $\langle\cdot, \cdot\rangle$. The Hilbert space adjoint for a linear operator $A$ with dense domain in $H$ is denoted by $A^{*}$.

\section{Background on polynomially stable semigroups}

In this section, we review the definition and some important properties of polynomially stable $C_{0}$-semigroups.

Definition 2.1. Let $\alpha>0$. A $C_{0}$-semigroup $(T(t))_{t \geq 0}$ on a Banach space $X$ generated by $A: D(A) \subset X \rightarrow X$ is polynomially stable with parameter $\alpha$ if $(T(t))_{t \geq 0}$ is uniformly bounded, $i \mathbb{R} \subset \varrho(A)$, and

$$
\left\|T(t) A^{-1}\right\|=O\left(\frac{1}{t^{1 / \alpha}}\right) \quad t \rightarrow \infty .
$$

The spectrum of the generator of any uniformly bounded semigroup is contained in the closed left half plane $\overline{\mathbb{C}_{-}}$. Therefore, if $A$ generates a polynomially stable semigroup, then $\sigma(A) \subset \mathbb{C}_{-}$.

Polynomial decay (2.1) of a $C_{0}$-semigroup $(T(t))_{t \geq 0}$ on a Hilbert space can be characterized by orbits as well; see [6, Theorem 2.4].

Theorem 2.2. Let $(T(t))_{t \geq 0}$ be a uniformly bounded $C_{0}$-semigroup on a Hilbert space $H$ with generator $A$ such that $i \mathbb{R} \subset \varrho(A)$. For a fixed $\alpha>0$, (2.1) holds if and only if

$$
\left\|T(t) A^{-1} x\right\|=o\left(\frac{1}{t^{1 / \alpha}}\right) \quad t \rightarrow \infty
$$

holds for every $x \in H$. 
For the generator $A$ of a polynomially stable $C_{0}$-semigroup, $-A$ is sectorial in the sense of [18, Chapter 2], and hence, the fractional powers $(-A)^{\alpha}$ are well defined for all $\alpha \in \mathbb{R}$. Using the moment inequality (see, e.g., Proposition 6.6.4 of [18]), we can normalize the decay rate in (2.1). See [2, Proposition 3.1] for the proof.

Lemma 2.3. Let $\alpha>0$ and $(T(t))_{t \geq 0}$ be a uniformly bounded $C_{0}$-semigroup on a Banach space with generator $A$ such that $0 \in \varrho(A)$. Then

$$
\left\|T(t)(-A)^{-\alpha}\right\|=O\left(\frac{1}{t}\right) \quad t \rightarrow \infty
$$

if and only if

$$
\left\|T(t)(-A)^{-\alpha \gamma}\right\|=O\left(\frac{1}{t^{\gamma}}\right) \quad t \rightarrow \infty
$$

for somelall $\gamma>0$.

A similar normalization result holds also for the case of orbits (2.2). The proof is essentially same as that of Lemma 2.3, i.e., it is a consequence of the moment inequality as stated in the proof of Theorem 2.4 of [6]. However, to make our presentation selfcontained, we give a short argument.

Lemma 2.4. Let $\alpha>0$ and $(T(t))_{t \geq 0}$ be a uniformly bounded $C_{0}$-semigroup on a Banach space $X$ with generator $A$ such that $0 \in \varrho(A)$. Then

$$
\left\|T(t)(-A)^{-\alpha} x\right\|=o\left(\frac{1}{t}\right) \quad t \rightarrow \infty
$$

for all $x \in X$ if and only if

$$
\left\|T(t)(-A)^{-\alpha \gamma} x\right\|=o\left(\frac{1}{t^{\gamma}}\right) \quad t \rightarrow \infty
$$

for all $x \in X$ and some/all $\gamma>0$.

Proof. Suppose that (2.4) holds for all $x \in X$ and some $\gamma>0$. Define $\delta:=\alpha \gamma$. Since

$$
\sup _{t \geq 0}\left\|t^{\gamma} T(t)(-A)^{-\delta} x\right\|<\infty
$$

for every $x \in X$, it follows from the uniform boundedness principle that there exists $C>0$ such that

$$
\sup _{t \geq 0}\left\|t^{\gamma} T(t)(-A)^{-\delta}\right\| \leq C .
$$

Take $x \in X, \varepsilon>0$ and $k \in \mathbb{N}$. Let

$$
0<\varepsilon_{0}<\frac{\varepsilon}{k^{k \gamma} C^{k-1}} .
$$


By (2.4), there exists $t_{0}>0$ such that

$$
\left\|T(t)(-A)^{-\delta} x\right\| \leq \frac{\varepsilon_{0}}{t^{\gamma}} \quad \forall t \geq t_{0} .
$$

For every $t \geq k t_{0}$,

$$
\begin{aligned}
\left\|T(t)(-A)^{-k \delta} x\right\| & \leq\left\|T(t / k)(-A)^{-\delta}\right\|^{k-1}\left\|T(t / k)(-A)^{-\delta} x\right\| \\
& \leq \frac{C^{k-1}}{(t / k)^{(k-1) \gamma}} \cdot \frac{\varepsilon_{0}}{(t / k)^{\gamma}} \\
& <\frac{\varepsilon}{t^{k \gamma}} .
\end{aligned}
$$

This implies that for every $k \in \mathbb{N}$,

$$
\left\|T(t)(-A)^{-k \delta} x\right\|=o\left(\frac{1}{t^{k \gamma}}\right) \quad t \rightarrow \infty .
$$

By the moment inequality, for every $k \in \mathbb{N}$ and every $\vartheta \in(0,1)$, there exists a constant $L_{1}>0$ such that

$$
\begin{aligned}
\left\|T(t)(-A)^{-k \delta \vartheta} x\right\| & =\left\|(-A)^{k \delta(1-\vartheta)} T(t)(-A)^{-k \delta} x\right\| \\
& \leq L_{1}\left\|(-A)^{k \delta} T(t)(-A)^{-k \delta} x\right\|^{1-\vartheta}\left\|T(t)(-A)^{-k \delta} x\right\|^{\vartheta} \\
& \leq L_{1} M^{1-\vartheta}\left\|T(t)(-A)^{-k \delta} x\right\|^{\vartheta}
\end{aligned}
$$

for all $t \geq 0$, where $M:=\sup _{t \geq 0}\|T(t) x\|$. This and (2.5) yield

$$
\left\|T(t)(-A)^{-k \delta \vartheta} x\right\|=o\left(\frac{1}{t^{k \gamma \vartheta}}\right) \quad t \rightarrow \infty .
$$

Setting $\vartheta=1 /(k \gamma)$ with $k>1 / \gamma$, we obtain (2.3).

Suppose that (2.3) holds for all $x \in X$. Take $\tilde{\gamma}>0$ and $x \in X$. Substituting $\gamma=1$ into (2.5), we have that for every $k \in \mathbb{N}$,

$$
\left\|T(t)(-A)^{-k \alpha} x\right\|=o\left(\frac{1}{t^{k}}\right) \quad t \rightarrow \infty .
$$

As in (2.6), we see that for every $k \in \mathbb{N}$ and every $\vartheta \in(0,1)$, there exists $L_{2}>0$ such that

$$
\left\|T(t)(-A)^{-k \alpha \vartheta} x\right\| \leq L_{2} M^{1-\vartheta}\left\|T(t)(-A)^{-k \alpha} x\right\|^{\vartheta} \quad \forall t \geq 0
$$

where $M:=\sup _{t \geq 0}\|T(t) x\|$. Setting $\vartheta=\tilde{\gamma} / k$ with $k>\tilde{\gamma}$, we obtain (2.4) with $\gamma=\tilde{\gamma}$.

In Lemma 2.4, we consider the global conditions on the decay of all orbits $\left\{(T(t) x)_{t \geq 0}: x \in X\right\}$. For individual orbits, a partial result holds. Since it can be obtained from the moment inequality as in (2.6), we omit the proof. 
Lemma 2.5. Let $\alpha, \beta>0$ and $(T(t))_{t \geq 0}$ be a uniformly bounded $C_{0}$-semigroup on a Banach space $X$ with generator $A$ such that $0 \in \varrho(A)$. If $x \in X$ satisfies

$$
\left\|T(t)(-A)^{-\alpha} x\right\|=o\left(\frac{1}{t^{\beta}}\right) \quad t \rightarrow \infty,
$$

then

$$
\left\|T(t)(-A)^{-\alpha \gamma} x\right\|=o\left(\frac{1}{t^{\beta \gamma}}\right) \quad t \rightarrow \infty
$$

holds for all $\gamma \in(0,1)$.

\section{Polynomial stability and Lyapunov equation}

In this section, we connect polynomial stability to a certain Lyapunov equation. To this end, we first develop an integral condition on resolvents for polynomial stability.

Proposition 3.1. Let $Q(\xi) \in \mathcal{L}(H)$ be a uniformly bounded $C_{0}$-semigroup on a Hilbert space $H$ with generator $A$ such that $i \mathbb{R} \subset \varrho(A)$. The following three assertions hold for a fixed $\alpha>0$ :

(a) $\left\|T(t)(-A)^{-\alpha}\right\|=O(1 / t)$ as $t \rightarrow \infty$ if and only if

$$
\lim _{\xi \rightarrow 0+} \xi^{1-2 \gamma} \int_{-\infty}^{\infty}\left\|R(\xi+i \eta, A)(-A)^{-\alpha \gamma} x\right\|^{2} d \eta=0
$$

for all $x \in H$ and somelall $\gamma \in(0,1 / 2)$.

(b) If $\left\|T(t)(-A)^{-\alpha}\right\|=O(1 / t)$ as $t \rightarrow \infty$, then

$$
\lim _{\xi \rightarrow 0+} \frac{1}{\log (1 / \xi)} \int_{-\infty}^{\infty}\left\|R(\xi+i \eta, A)(-A)^{-\alpha / 2} x\right\|^{2} d \eta=0
$$

for all $x \in H$.

(c) If (3.2) holds for all $x \in H$, then

$$
\left\|T(t)(-A)^{-\alpha}\right\|=O\left(\frac{\log t}{t}\right) \quad t \rightarrow \infty .
$$

To prove Proposition 3.1, we study the decay rate of an individual orbit.

Lemma 3.2. Let $(T(t))_{t \geq 0}$ be a uniformly bounded $C_{0}$-semigroup on a Hilbert space $H$ with generator $A$ such that $0 \in \varrho(A)$. The following two assertions hold for fixed $\alpha>0$ and $x \in H$ :

(a) If $\left\|T(t)(-A)^{-\alpha} x\right\|=o(1 / t)$ as $t \rightarrow \infty$, then

$$
\lim _{\xi \rightarrow 0+} \xi^{1-2 \gamma} \int_{-\infty}^{\infty}\left\|R(\xi+i \eta, A)(-A)^{-\alpha \gamma} x\right\|^{2} d \eta=0
$$

for all $\gamma \in(0,1 / 2)$ and

$$
\lim _{\xi \rightarrow 0+} \frac{1}{\log (1 / \xi)} \int_{-\infty}^{\infty}\left\|R(\xi+i \eta, A)(-A)^{-\alpha / 2} x\right\|^{2} d \eta=0 .
$$


(b) If (3.4) holds for some $\gamma \in(0,1 / 2)$, then

$$
\left\|T(t)(-A)^{-\alpha \gamma} x\right\|=o\left(\frac{1}{t \gamma}\right) \quad t \rightarrow \infty .
$$

On the other hand, if (3.5) holds, then

$$
\left\|T(t)(-A)^{-\alpha / 2} x\right\|=o\left(\sqrt{\frac{\log t}{t}}\right) \quad t \rightarrow \infty .
$$

Proof. (a) Suppose that $x \in H$ satisfies $\left\|T(t)(-A)^{-\alpha} x\right\|=o(1 / t)$ as $t \rightarrow \infty$. Let $\varepsilon>0$ and $\gamma \in(0,1 / 2]$. By Lemma 2.5, there exists $t_{0}>0$ such that for all $t \geq t_{0}$,

$$
\left\|T(t)(-A)^{-\alpha \gamma} x\right\| \leq \frac{\varepsilon}{t^{\gamma}} .
$$

By the Plancherel theorem,

$$
\frac{1}{2 \pi} \int_{-\infty}^{\infty}\|R(\xi+i \eta, A) x\|^{2} d \eta=\int_{0}^{\infty} e^{-2 \xi t}\|T(t) x\|^{2} d t
$$

for all $\xi>0$. Using (3.8), we obtain

$$
\begin{aligned}
& \int_{0}^{\infty} e^{-2 \xi t}\left\|T(t)(-A)^{-\alpha \gamma} x\right\|^{2} d t \\
& \quad=\int_{0}^{t_{0}} e^{-2 \xi t}\left\|T(t)(-A)^{-\alpha \gamma} x\right\|^{2} d t+\int_{t_{0}}^{\infty} e^{-2 \xi t}\left\|T(t)(-A)^{-\alpha \gamma} x\right\|^{2} d t \\
& \leq t_{0} M^{2}\|x\|^{2}+\varepsilon^{2} \int_{t_{0}}^{\infty} \frac{e^{-2 \xi t}}{t^{2 \gamma}} d t
\end{aligned}
$$

for all $\xi>0$, where $M:=\left\|(-A)^{-\alpha \gamma}\right\| \sup _{t \geq 0}\|T(t)\|$.

First we consider the case $\gamma \in(0,1 / 2)$. We have that

$$
\int_{0}^{\infty} \frac{e^{-2 \xi t}}{t^{2 \gamma}} d t=\frac{\Gamma(1-2 \gamma)}{(2 \xi)^{1-2 \gamma}}
$$

for all $\xi>0$, where $\Gamma$ is the gamma function. Hence, (3.9) yields

$$
\limsup _{\xi \rightarrow 0+} \xi^{1-2 \gamma} \int_{0}^{\infty} e^{-2 \xi t}\left\|T(t)(-A)^{-\alpha \gamma} x\right\|^{2} d t \leq \frac{\Gamma(1-2 \gamma) \varepsilon^{2}}{2^{1-2 \gamma}} .
$$

Since $\varepsilon>0$ was arbitrary, it follows that (3.4) holds.

Next we investigate the case $\gamma=1 / 2$. By the inequality (5) of [11], the exponential integral satisfies

$$
\int_{\tau}^{\infty} \frac{e^{-t}}{t} d t \leq e^{-\tau} \log \left(1+\frac{1}{\tau}\right)
$$


for all $\tau>0$. Hence,

$$
\int_{t_{0}}^{\infty} \frac{e^{-2 \xi t}}{t} d t=\int_{2 \xi t_{0}}^{\infty} \frac{e^{-t}}{t} d t \leq e^{-2 \xi t_{0}} \log \left(1+\frac{1}{2 \xi t_{0}}\right)
$$

for every $\xi>0$. Applying this estimate to (3.9), we obtain

$$
\limsup _{\xi \rightarrow 0+} \frac{1}{\log \left(1+\frac{1}{2 \xi t_{0}}\right)} \int_{0}^{\infty} e^{-2 \xi t}\left\|T(t)(-A)^{-\alpha / 2} x\right\|^{2} d t \leq \varepsilon^{2} .
$$

There exists $0<\xi_{0}<1$ such that for all $\xi \in\left(0, \xi_{0}\right)$,

$$
\log \left(1+\frac{1}{2 \xi t_{0}}\right) \leq 2 \log (1 / \xi)
$$

Since $\varepsilon>0$ was arbitrary, it follows from (3.10) that (3.5) holds.

b) By Theorem I.3.9 and Proposition I.3.10 of [9], we have that

$$
T(t) x=\frac{1}{2 \pi t} \int_{-\infty}^{\infty} e^{(\xi+i \eta) t} R(\xi+i \eta, A)^{2} x d \eta
$$

for all $x \in H, \xi>0$ and $t>0$. The Cauchy-Schwartz inequality gives

$$
\begin{aligned}
|\langle T(t) x, y\rangle| & \leq \frac{e^{\xi t}}{2 \pi t} \int_{-\infty}^{\infty}\left|\left\langle R(\xi+i \eta, A) x, R\left(\xi-i \eta, A^{*}\right) y\right\rangle\right| d \eta \\
& \leq \frac{e^{\xi t}}{2 \pi t}\left(\int_{-\infty}^{\infty}\|R(\xi+i \eta, A) x\|^{2} d \eta\right)^{\frac{1}{2}}\left(\int_{-\infty}^{\infty}\left\|R\left(\xi+i \eta, A^{*}\right) y\right\|^{2} d \eta\right)^{\frac{1}{2}}
\end{aligned}
$$

for all $x, y \in H, \xi>0$ and $t>0$. Since $(T(t))_{t \geq 0}$ is uniformly bounded, it follows from the Plancherel theorem that

$$
\begin{aligned}
\sup _{\xi>0} \xi \int_{-\infty}^{\infty}\left\|R\left(\xi+i \eta, A^{*}\right) y\right\|^{2} d \eta & =2 \pi \sup _{\xi>0} \xi \int_{0}^{\infty} e^{-2 \xi t}\left\|T(t)^{*} y\right\|^{2} d \eta \\
& \leq \pi M^{2}\|y\|^{2}
\end{aligned}
$$

for every $y \in H$, where $M:=\sup _{t \geq 0}\|T(t)\|=\sup _{t \geq 0}\left\|T(t)^{*}\right\|$. This and (3.11) establish

$$
\|T(t) x\| \leq \frac{M e^{\xi t}}{2 t \sqrt{\pi \xi}}\left(\int_{-\infty}^{\infty}\|R(\xi+i \eta, A) x\|^{2} d \eta\right)^{\frac{1}{2}}
$$

for all $x \in H, \xi>0$ and $t>0$.

Suppose that $x \in H$ satisfies (3.4) for some $0<\gamma<1 / 2$ or (3.5). In the latter case, we set $\gamma=1 / 2$. For $\xi>0$, define

$$
h_{\gamma}(\xi):= \begin{cases}\xi^{1-2 \gamma} & \text { if } 0<\gamma<1 / 2 \\ \frac{1}{\log (1 / \xi)} & \text { if } \gamma=1 / 2\end{cases}
$$


Taking $\xi=1 / t$, we have from (3.12) that for every $\varepsilon>0$, there exists $t_{0}>1$ such that for all $t \geq t_{0}$,

$$
\left\|T(t)(-A)^{-\alpha \gamma} x\right\| \leq \frac{M e \varepsilon}{2 \sqrt{\pi}} \sqrt{\frac{1}{t h_{\gamma}(1 / t)}} .
$$

By the definition (3.13) of $h_{\gamma}$, we obtain (3.6) for $0<\gamma<1 / 2$ and (3.7) for $\gamma=1 / 2$.

Proof of Proposition 3.1. We easily see that the assertions a) and b) hold, by combining Theorem 2.2 with Lemmas 2.3, 2.4 and 3.2 .

It remains to prove that (c) holds. By (b) of Lemma 3.2,

$$
\sup _{t>2} \sqrt{\frac{t}{\log t}}\left\|T(t)(-A)^{-\alpha / 2} x\right\|<\infty
$$

for every $x \in H$. By the uniform boundedness principle, there exists $C_{1}>0$ such that

$$
\left\|T(t)(-A)^{-\alpha / 2}\right\| \leq C_{1} \sqrt{\frac{\log t}{t}} \quad \forall t>2 .
$$

Hence,

$$
\begin{aligned}
\left\|T(t)(-A)^{-\alpha}\right\| & \leq\left\|T(t / 2)(-A)^{-\alpha / 2}\right\|^{2} \\
& \leq C_{1}^{2} \frac{\log (t / 2)}{t / 2} \quad \forall t>4
\end{aligned}
$$

Thus, (3.3) holds.

Let $\xi>0$ and $A$ be the generator of a uniformly bounded $C_{0}$-semigroup $(T(t))_{t \geq 0}$ on a Hilbert space $H$. Consider the Lyapunov equation

$$
\left\langle(A-\xi I) x_{1}, Q(\xi) x_{2}\right\rangle+\left\langle Q(\xi) x_{1},(A-\xi I) x_{2}\right\rangle=-\left\langle x_{1}, x_{2}\right\rangle
$$

for $x_{1}, x_{2} \in D(A)$. It has a unique self-adjoint solution $Q(\xi) \in \mathcal{L}(H)$ given by

$$
Q(\xi) x:=\int_{0}^{\infty} e^{-2 \xi t} T(t)^{*} T(t) x d t, \quad x \in H ;
$$

see, e.g., Theorem 4.1.23 of [8].

We restate Proposition 3.1 by using the self-adjoint solution of the Lyapunov Eq. (3.14).

Lemma 3.3. Let $(T(t))_{t \geq 0}$ be a uniformly bounded $C_{0}$-semigroup on a Hilbert space $H$ with generator $A$ such that $i \mathbb{R} \subset \varrho(A)$, and let $Q(\xi) \in \mathcal{L}(H)$ be the self-adjoint solution of the Lyapunov Eq. (3.14) for $\xi>0$. The following three assertions hold for a fixed $\alpha>0$ : 
(a) $\left\|T(t)(-A)^{-\alpha}\right\|=O(1 / t)$ as $t \rightarrow \infty$ if and only if $Q(\xi)$ satisfies

$$
\lim _{\xi \rightarrow 0+} \xi^{1-2 \gamma}\left\langle(-A)^{-\alpha \gamma} x, Q(\xi)(-A)^{-\alpha \gamma} x\right\rangle=0
$$

for all $x \in H$ and some/all $\gamma \in(0,1 / 2)$.

(b) If $\left\|T(t)(-A)^{-\alpha}\right\|=O(1 / t)$ as $t \rightarrow \infty$, then $Q(\xi)$ satisfies

$$
\lim _{\xi \rightarrow 0+} \frac{\left\langle(-A)^{-\alpha / 2} x, Q(\xi)(-A)^{-\alpha / 2} x\right\rangle}{\log (1 / \xi)}=0
$$

for all $x \in H$.

(c) If $Q(\xi)$ satisfies (3.17) for all $x \in H$, then

$$
\left\|T(t)(-A)^{-\alpha}\right\|=O\left(\frac{\log t}{t}\right) \quad t \rightarrow \infty .
$$

Proof. Let $\xi>0$ and $x \in H$. The Plancherel theorem shows that

$$
\frac{1}{2 \pi} \int_{-\infty}^{\infty}\|R(\xi+i \eta, A) x\|^{2} d \eta=\int_{0}^{\infty} e^{-2 \xi t}\|T(t) x\|^{2} d t .
$$

By the definition (3.15) of $Q(\xi)$, we obtain

$$
\langle x, Q(\xi) x\rangle=\frac{1}{2 \pi} \int_{-\infty}^{\infty}\|R(\xi+i \eta, A) x\|^{2} d \eta .
$$

Hence, the assertions (a)-(c) immediately follow from Proposition 3.1.

\section{Decay rate of Cayley transform}

In this section, we study the decay rate of the Cayley transform of the generator of a polynomially stable $C_{0}$-semigroup. We start by establishing the discrete version of the normalization results on polynomial decay rates developed in Lemmas 2.3-2.5.

Lemma 4.1. Let $A$ be the generator of a uniformly bounded $C_{0}$-semigroup on a Banach space $X$ such that $0 \in \varrho(A)$. Suppose that $B \in \mathcal{L}(X)$ is power bounded and commutes with $A^{-1}$. Then the following assertions hold for constants $\alpha, \beta>0$ and $a$ nondecreasing function $f: \mathbb{N} \rightarrow(0, \infty)$.

(a) The statements (i)-(ii) below are equivalent:

(i) $\left\|B^{n}(-A)^{-\alpha}\right\|=O\left(\frac{f(n)}{n}\right)$ as $n \rightarrow \infty$.

(ii) $\left\|B^{n}(-A)^{-\alpha \gamma}\right\|=O\left(\frac{f(n)^{\gamma}}{n^{\gamma}}\right)$ as $n \rightarrow \infty$ for somelall $\gamma>0$.

(b) The statements (i)-(ii) below are equivalent:

(i) $\left\|B^{n}(-A)^{-\alpha} x\right\|=o\left(\frac{f(n)}{n}\right)$ as $n \rightarrow \infty$ for all $x \in X$. 
(ii) $\left\|B^{n}(-A)^{-\alpha \gamma} x\right\|=o\left(\frac{f(n)^{\gamma}}{n^{\gamma}}\right)$ as $n \rightarrow \infty$ for all $x \in X$ and somelall $\gamma>0$.

(c) If $x \in X$ satisfies

$$
\left\|B^{n}(-A)^{-\alpha} x\right\|=o\left(\frac{f(n)^{\beta}}{n^{\beta}}\right) \quad n \rightarrow \infty,
$$

then

$$
\left\|B^{n}(-A)^{-\alpha \gamma} x\right\|=o\left(\frac{f(n)^{\beta \gamma}}{n^{\beta \gamma}}\right) \quad n \rightarrow \infty
$$

for every $0<\gamma<1$.

Proof. (a) (ii) $\Rightarrow$ (i): Let $\gamma>0$ and set $\delta:=\alpha \gamma$. By assumption, there exists a constant $C>0$ such that

$$
\left\|B^{n}(-A)^{-\delta}\right\| \leq C\left(\frac{f(n)}{n}\right)^{\gamma} \quad \forall n \in \mathbb{N} .
$$

For every $a>0, B$ commutes with $(-A)^{-a}$ by Proposition 3.1.1.f) of [18], and hence, for every $x \in D\left((-A)^{a}\right), B x \in D\left((-A)^{a}\right)$ and $B(-A)^{a} x=(-A)^{a} B x$ by Proposition B.7 of [1]. Using (4.1), we obtain

$$
\left\|B^{k n}(-A)^{-k \delta}\right\| \leq\left\|B^{n}(-A)^{-\delta}\right\|^{k} \leq C^{k}\left(\frac{f(n)}{n}\right)^{k \gamma} \quad \forall k, n \in \mathbb{N} .
$$

From the moment inequality (see, e.g., Proposition 6.6.4 of [18]), it follows that for every $k \in \mathbb{N}$ and every $\vartheta \in(0,1)$, there exists a constant $L_{0}>0$ such that

$$
\begin{aligned}
\left\|B^{k n}(-A)^{-\vartheta k \delta} x\right\| & =\left\|(-A)^{k \delta(1-\vartheta)} B^{k n}(-A)^{-k \delta} x\right\| \\
& \leq L_{0}\left\|(-A)^{k \delta} B^{k n}(-A)^{-k \delta} x\right\|^{1-\vartheta}\left\|B^{k n}(-A)^{-k \delta} x\right\|^{\vartheta} \\
& \leq L_{0}\left\|B^{k n}\right\|^{1-\vartheta}\left\|B^{k n}(-A)^{-k \delta}\right\|^{\vartheta}\|x\|
\end{aligned}
$$

for every $x \in X$ and every $n \in \mathbb{N}$. Hence, (4.2) yields

$$
\left\|B^{k n}(-A)^{-\vartheta k \delta}\right\| \leq L_{0} M^{1-\vartheta} C^{k \vartheta}\left(\frac{f(n)}{n}\right)^{k \gamma \vartheta} \quad \forall n \in \mathbb{N},
$$

where $M:=\sup _{n \in \mathbb{N} \cup\{0\}}\left\|B^{n}\right\|$. Choose a constant $k \in \mathbb{N}$ satisfying $k>1 / \gamma$ and set $\vartheta=1 /(k \gamma)$. Then

$$
\left\|B^{k n}(-A)^{-\alpha}\right\| \leq k L_{1} M C^{1 / \gamma} \frac{f(k n)}{k n} \quad \forall n \in \mathbb{N}
$$

for some $L_{1}>0$. Since

$$
\frac{k n+\ell}{k n} \leq \frac{k(n+1)}{k n} \leq 2
$$


for every $\ell=0, \ldots, k-1$ and every $n \in \mathbb{N}$, it follows that

$$
\begin{aligned}
\left\|B^{k n+\ell}(-A)^{-\alpha}\right\| & \leq\left\|B^{\ell}\right\|\left\|B^{k n}(-A)^{-\alpha}\right\| \\
& \leq 2 k L_{1} M^{2} C^{1 / \gamma} \frac{f(k n+\ell)}{k n+\ell}
\end{aligned}
$$

for every $\ell=0, \ldots, k-1$ and every $n \in \mathbb{N}$. Thus, (i) holds.

(i) $\Rightarrow$ (ii): Take $\tilde{\gamma}>0$. Substituting $\gamma=1$ and $\vartheta=\tilde{\gamma} / k$ with $k>\tilde{\gamma}$ into (4.4), we obtain

$$
\left\|B^{k n}(-A)^{-\alpha \tilde{\gamma}}\right\| \leq L_{2} M C^{\tilde{\gamma}}\left(\frac{f(n)}{n}\right)^{\tilde{\gamma}} \quad \forall n \in \mathbb{N}
$$

for some $L_{2}>0$. Hence, we obtain (ii) with $\gamma=\tilde{\gamma}$ by similar arguments as above.

(b) Suppose that (ii) holds, and set $\delta:=\alpha \gamma$. Since

$$
\sup _{n \in \mathbb{N}}\left(\frac{n}{f(n)}\right)^{\gamma}\left\|B^{n}(-A)^{-\delta} x\right\|<\infty,
$$

for all $x \in X$, it follows from the uniform boundedness principle that there exists a constant $C_{1}>0$ such that

$$
\left\|B(-A)^{-\delta}\right\| \leq C_{1}\left(\frac{f(n)}{n}\right)^{\gamma} \quad \forall n \in \mathbb{N} .
$$

Let $x \in X$. Then

$$
\begin{aligned}
\left\|B^{k n}(-A)^{-k \delta} x\right\| & \leq\left\|B^{n}(-A)^{-\delta}\right\|^{k-1}\left\|B^{n}(-A)^{-\delta} x\right\| \\
& \leq C_{1}^{k-1}\left(\frac{f(n)}{n}\right)^{(k-1) \gamma}\left\|B^{n}(-A)^{-\delta} x\right\| \quad \forall k, n \in \mathbb{N} .
\end{aligned}
$$

This implies

$$
\left\|B^{k n}(-A)^{-k \delta} x\right\|=o\left(\frac{f(n)^{k \gamma}}{n^{k \gamma}}\right) \quad n \rightarrow \infty .
$$

The rest of the proof is the same as the proof of (a). We omit the details.

(c) This assertion directly follows from the application of the moment inequality as in (4.3).

Using the argument based on Lyapunov equations developed in Sect. 3, we estimate the decay rate of the Cayley transform. We use the following preliminary result obtained in the proof of [17, Theorem 4.3].

Lemma 4.2. Let $A$ be the generator of a uniformly bounded $C_{0}$-semigroup on a Hilbert space $H$. Suppose that the Cayley transform $A_{d}:=(I+A)(I-A)^{-1}$ is power bounded. Let $r \in(0,1)$ and

$$
2 \xi=\frac{1-r^{2}}{1+r^{2}} .
$$


Then the self-adjoint solution $Q(\xi) \in \mathcal{L}(H)$ of the Lyapunov Eq. (3.14) satisfies

$$
(n+1)\left|\left\langle y, r^{n} A_{d}^{n}(I-A)^{-1} x\right\rangle\right| \leq M\|y\| \sqrt{\frac{\langle x, Q(\xi) x\rangle}{1-r^{4}}}
$$

for every $x, y \in H$ and every $n \in \mathbb{N}$, where $M:=\sup _{n \in \mathbb{N} \cup\{0\}}\left\|A_{d}^{n}\right\|$.

Theorem 4.3. Let $(T(t))_{t \geq 0}$ be a uniformly bounded $C_{0}$-semigroup on a Hilbert space $H$ with generator $A$ such that $i \mathbb{R} \subset \varrho(A)$. Suppose that the Cayley transform $A_{d}:=$ $(I+A)(I-A)^{-1}$ is power bounded. If $\left\|T(t)(-A)^{-\alpha}\right\|=O(1 / t)$ as $t \rightarrow \infty$ for some $\alpha>0$, then $A_{d}$ satisfies

$$
\left\|A_{d}^{n}(-A)^{-\alpha-2} x\right\|=o\left(\frac{\log n}{n}\right) \quad n \rightarrow \infty
$$

for every $x \in H$ and

$$
\left\|A_{d}^{n}(-A)^{-\alpha-2}\right\|=O\left(\frac{\log n}{n}\right) \quad n \rightarrow \infty .
$$

Proof. Take $r \in(0,1)$. Let $Q(\xi) \in \mathcal{L}(H)$ be given in (4.5) and $Q(\xi)$ be the selfadjoint solution of the Lyapunov Eq. (3.14). By Lemma 4.2, $Q(\xi)$ satisfies (4.6) for every $x, y \in H$ and every $n \in \mathbb{N}$.

Let $\varepsilon>0, y \in H$ and $x \in D\left((-A)^{\alpha / 2}\right)$. Define $\tilde{x}:=(I-A)^{-1} x$. By Lemma 3.3(b), there exists $N_{0} \in \mathbb{N}$ such that

$$
\frac{\langle x, Q(\xi) x\rangle}{\log (1 / \xi)} \leq \varepsilon^{2}
$$

for

$$
2 \xi=\frac{2 n+1}{2 n^{2}+2 n+1}
$$

with $n \geq N_{0}$. For $\xi$ given in (4.9),

$$
r=\frac{n}{n+1}
$$

satisfies (4.5) and

$$
\frac{\log (1 / \xi)}{1-r^{4}} \leq C_{1}^{2} n \log n \quad \forall n \in \mathbb{N}
$$

for some constant $C_{1}>0$. Therefore, (4.6) yields

$$
\left|\left\langle y, A_{d}^{n} \tilde{x}\right\rangle\right| \leq M C_{1}\|y\| \varepsilon \cdot \frac{\sqrt{n \log n}}{n+1}\left(1+\frac{1}{n}\right)^{n} \quad \forall n \geq N_{0} .
$$

This implies that

$$
\left\|A_{d}^{n} \tilde{x}\right\|=o\left(\sqrt{\frac{\log n}{n}}\right) \quad n \rightarrow \infty
$$


Since

$$
\left\{\tilde{x}=(I-A)^{-1} x: x \in D\left((-A)^{\alpha / 2}\right)\right\}=\operatorname{ran}\left((-A)^{-\alpha / 2-1}\right),
$$

we obtain

$$
\left\|A_{d}^{n}(-A)^{-\alpha / 2-1} x\right\|=o\left(\sqrt{\frac{\log n}{n}}\right) \quad n \rightarrow \infty
$$

for every $x \in H$. The first assertion (4.7) follows from Lemma 4.1. Applying the uniform boundedness principle to (4.7), we obtain (4.8).

Remark 4.4. In the Proof of Theorem 4.3, we employ (b) of Lemma 3.3. One can apply (a) of Lemma 3.3 in a similar way and consequently obtain

$$
\left\|A_{d}^{n}(-A)^{-\alpha-1 / \gamma}\right\|=O\left(\frac{1}{n}\right) \quad n \rightarrow \infty
$$

for every $0<\gamma<1 / 2$. However, this result is less sharp than (4.8).

We do not know whether the logarithmic factor in Theorem 4.3 may be dropped in general. In some cases, however, we can omit the logarithmic correction, as in Propositions 4.1 and 4.2 in [2]. It is worth mentioning that we do not need the assumption on the power boundedness of the Cayley transform.

Proposition 4.5. Consider the following two cases:

(a) Let $(T(t))_{t \geq 0}$ be a uniformly bounded $C_{0}$-semigroup on a Hilbert space with generator $A$ such that $i \mathbb{R} \subset \varrho(A)$ and $A$ is normal.

(b) Let $\Omega$ be a locally compact Hausdorff space and $\mu$ be a $\sigma$-finite regular Borel measure on $\Omega$. Assume that either

(i) $X:=L^{p}(\Omega, \mu)$ for $1 \leq p<\infty$ and $\phi: \Omega \rightarrow \mathbb{C}$ is measureable with essential range in the open left half plane $\mathbb{C}_{-}$; or that

(ii) $X:=C_{0}(\Omega)$ and $\phi: \Omega \rightarrow \mathbb{C}$ is continuous with $\overline{\phi(\Omega)} \subset \mathbb{C}_{-}$.

Let $A$ be the multiplication operator induced by $\phi$ on $X$, i.e, Af $=\phi f$ with domain $D(A):=\{f \in X: \phi f \in X\}$ and $(T(t))_{t \geq 0}$ be the $C_{0}$-semigroup on $X$ generated by $A$.

In both cases (a) and (b), if $\left\|T(t)(-A)^{-\alpha}\right\|=O(1 / t)$ as $t \rightarrow \infty$ for some $\alpha>0$, then the Cayley transform $A_{d}:=(I+A)(I-A)^{-1}$ satisfies

$$
\left\|A_{d}^{n}(-A)^{-\alpha-2}\right\|=O\left(\frac{1}{n}\right) \quad n \rightarrow \infty .
$$

Proof. The proof is divided up into two steps. In the first step, we characterize the norm of $A_{d}^{n}(-A)^{-\alpha}$ for $n \in \mathbb{N}$ by the spectrum of $A$. In the second step, we obtain the decay estimate (4.10) from this characterization and the geometrical condition on $\sigma(-A)$ for polynomial decay given in Propositions 4.1 and 4.2 of [2]. 
Step 1: First, we consider the case (a). Define

$$
f_{n}(\lambda):=\left(\frac{1-\lambda}{1+\lambda}\right)^{n} \lambda^{-\alpha-2}
$$

for $\lambda \in \mathbb{C} \backslash(-\infty, 0]$ and $n \in \mathbb{N}$. Then $A_{d}^{n}(-A)^{-\alpha-2}=f_{n}(-A)$ by the product formula of functional calculi (see, e.g., Theorem 1.3.2.c) of [18]). The spectral mapping theorem (see, e.g., Theorem 2.7 .8 of [18]) shows that

$$
\sigma\left(f_{n}(-A)\right)=f_{n}(\tilde{\sigma}(-A)),
$$

where $f_{n}(\infty):=\lim _{\lambda \rightarrow 0} f_{n}(1 / \lambda)=0$. Since $A$ is normal, we see that $f_{n}(-A)$ is also normal, by using a multiplication operator unitarily equivalent to $A$ obtained from the spectral theorem; see, for example, Theorem 4.1 of [19] for the multiplicator version of the spectral theorem for unbounded normal operators. Moreover, $f_{n}(-A)$ is bounded. Hence, the spectral radius of $f_{n}(-A)$ equals $\left\|f_{n}(-A)\right\|$; see, e.g., Theorem 5.44 of [34]. This yields

$$
\left\|A_{d}^{n}(-A)^{-\alpha-2}\right\|=\left\|f_{n}(-A)\right\|=\sup _{\lambda \in \sigma\left(f_{n}(-A)\right)}|\lambda|=\sup _{\lambda \in \tilde{\sigma}(-A)}\left|f_{n}(\lambda)\right| .
$$

A result similar to (4.12) is obtained in the case (b). Let $h_{\mathrm{ess}}(\Omega)$ denote the essential range of a measurable function $h: \Omega \rightarrow \mathbb{C}$. Define the function $f_{n}$ as in (4.11). In the $L^{p}$-case (i),

$$
\begin{aligned}
\left\|A_{d}^{n}(-A)^{-\alpha-2}\right\| & =\sup \left\{|z|: z \in\left(f_{n} \circ(-\phi)\right)_{\mathrm{ess}}(\Omega)\right\} \\
\sigma(A) & =\phi_{\mathrm{ess}}(\Omega)
\end{aligned}
$$

by Proposition I.4.10 of [10]. Moreover, one can obtain

$$
\sup \left\{|z|: z \in\left(f_{n} \circ(-\phi)\right)_{\mathrm{ess}}(\Omega)\right\}=\sup \left\{\left|f_{n}(\lambda)\right|:-\lambda \in \phi_{\mathrm{ess}}(\Omega)\right\} .
$$

Therefore,

$$
\left\|A_{d}^{n}(-A)^{-\alpha-2}\right\|=\sup _{\lambda \in \sigma(-A)}\left|f_{n}(\lambda)\right|
$$

In the $C_{0}$-case (ii), we also obtain (4.13), since

$$
\begin{aligned}
\left\|A_{d}^{n}(-A)^{-\alpha-2}\right\| & =\sup _{s \in \Omega}\left|\left(f_{n} \circ(-\phi)\right)(s)\right| \\
\sigma(A) & =\overline{\phi(\Omega)}
\end{aligned}
$$

by Proposition I.4.2 of [10].

Step 2: Propositions 4.1 and 4.2 of [2] show that in both cases (a) and (b), $\left\|T(t)(-A)^{-\alpha}\right\|=O(1 / t)$ as $t \rightarrow \infty$ if and only if there exist $\delta, C>0$ such that $|\operatorname{Im} \lambda| \geq C(\operatorname{Re} \lambda)^{-1 / \alpha}$ for every $\lambda \in \sigma(-A)$ with $\operatorname{Re} \lambda \leq \delta$. Define

$$
\Omega_{1}:=\{\lambda \in \sigma(-A): \operatorname{Re} \lambda \leq \delta\}, \quad \Omega_{2}:=\sigma(-A) \backslash \Omega_{1} .
$$


In what follows, we estimate $\left|n f_{n}(\lambda)\right|$ for $\lambda \in \sigma(-A)$, by dividing the argument into two cases: $\lambda \in \Omega_{1}$ and $\lambda \in \Omega_{2}$. To this end, we use the identities

$$
\left|\frac{1-\lambda}{1+\lambda}\right|^{n}=\left(\frac{(1-\operatorname{Re} \lambda)^{2}+|\operatorname{Im} \lambda|^{2}}{(1+\operatorname{Re} \lambda)^{2}+|\operatorname{Im} \lambda|^{2}}\right)^{\frac{n}{2}}=\left(1-\frac{4 \operatorname{Re} \lambda}{|1+\lambda|^{2}}\right)^{\frac{n}{2}}
$$

for $\lambda \in \sigma(-A)$.

First, we consider the case $\lambda \in \Omega_{1} \subset\left\{s \in \mathbb{C}:|\operatorname{Im} \lambda| \geq C(\operatorname{Re} \lambda)^{-1 / \alpha}\right\}$. Then

$$
1 \geq \frac{4 \operatorname{Re} \lambda}{|1+\lambda|^{2}} \geq \frac{\frac{4 C^{\alpha}}{|\operatorname{Im} \lambda|^{\alpha}}}{|1+\lambda|^{2}} \geq \frac{4 C^{\alpha}}{C_{1}|\operatorname{Im} \lambda|^{\alpha+2}}>0
$$

for some $C_{1} \geq 1$. Set $C_{2}:=4 C^{\alpha} / C_{1}$. Then

$$
n\left|f_{n}(\lambda)\right| \leq \frac{n}{|\operatorname{Im} \lambda|^{\alpha+2}}\left(1-\frac{C_{2}}{|\operatorname{Im} \lambda|^{\alpha+2}}\right)^{\frac{n}{2}}
$$

for all $n \in \mathbb{N}$. Define

$$
g_{n}(s):=\frac{n}{s}\left(1-\frac{C_{2}}{s}\right)^{\frac{n}{2}}
$$

for $s \geq C_{2}$ and $n \in \mathbb{N}$. Then

$$
g_{n}^{\prime}(s)=\frac{n\left(1-\frac{C_{2}}{s}\right)^{\frac{n}{2}-1}\left(C_{2}(n+2)-2 s\right)}{2 s^{3}} \quad \forall s>C_{2} .
$$

Hence, $g_{n}$ takes the maximum value at $s=s^{*}:=\frac{C_{2}(n+2)}{2}$ on $\left[C_{2}, \infty\right)$ and

$$
g_{n}\left(s^{*}\right)=\frac{2 n}{C_{2}(n+2)}\left(1-\frac{2}{n+2}\right)^{\frac{n}{2}} \rightarrow \frac{2}{e C_{2}} \quad n \rightarrow \infty .
$$

This means that

$$
\sup _{n \in \mathbb{N}} \sup _{\lambda \in \Omega_{1}} n\left|f_{n}(\lambda)\right|<\infty \text {. }
$$

Next we investigate the case $\lambda \in \Omega_{2}$. We have that

$$
1 \geq \frac{4 \operatorname{Re} \lambda}{|1+\lambda|^{2}} \geq \frac{4 \delta}{(1+|\lambda|)^{2}} \geq \frac{4 \delta}{(1+1 / \delta)^{2}|\lambda|^{2}}>0 .
$$

Set $C_{3}:=4 \delta /(1+1 / \delta)^{2}$. Then

$$
n\left|f_{n}(\lambda)\right| \leq \frac{1}{\delta^{\alpha}} \frac{n}{|\lambda|^{2}}\left(1-\frac{C_{3}}{|\lambda|^{2}}\right)^{\frac{n}{2}} .
$$

Similarly to the case $\lambda \in \Omega_{1}$, we obtain

$$
\sup _{n \in \mathbb{N}} \sup _{\lambda \in \Omega_{2}} n\left|f_{n}(\lambda)\right|<\infty \text {. }
$$


Combining (4.14) and (4.15), we have that

$$
\sup _{n \in \mathbb{N}} n\left\|A_{d}^{n}(-A)^{-\alpha-2}\right\|<\infty .
$$

Thus, the desired conclusion (4.10) holds.

Example 4.6. There exists a normal operator $A$ such that the norm estimate of Cayley transforms in Proposition 4.5 cannot be improved. Consider a diagonal operator $A$ : $D(A) \subset \ell^{2}(\mathbb{C}) \rightarrow \ell^{2}(\mathbb{C})$ defined by

$$
A x:=\sum_{k \in \mathbb{N}}\left(-\frac{1}{k}+i k\right)\left\langle x, e_{k}\right\rangle e_{k}
$$

with domain

$$
D(A):=\left\{x \in \ell^{2}(\mathbb{C}): \sum_{k \in \mathbb{N}} k^{2}\left|\left\langle x, e_{k}\right\rangle\right|^{2}<\infty\right\},
$$

where $\left(e_{k}\right)_{k \in \mathbb{N}}$ is the standard basis of $\ell^{2}(\mathbb{C})$. The semigroup $(T(t))_{t \geq 0}$ generated by $A$ satisfies $\left\|T(t) A^{-1}\right\|=O(1 / t)$ as $t \rightarrow \infty$ by Proposition 4.1 of [2]. We see from Proposition 4.5 that

$$
\left\|A_{d}^{n} A^{-3}\right\|=O\left(\frac{1}{n}\right) \quad n \rightarrow \infty .
$$

The Cayley transform $A_{d}$ is given by

$$
A_{d} x=\sum_{k \in \mathbb{N}} \frac{k-1+i k^{2}}{k+1-i k^{2}}\left\langle x, e_{k}\right\rangle e_{k} \quad \forall x \in X .
$$

Since

$$
\left|\frac{k-1+i k^{2}}{k+1-i k^{2}}\right|^{2}=\frac{(k-1)^{2}+k^{4}}{(k+1)^{2}+k^{4}} \leq 1
$$

for all $k \in \mathbb{N}$, it follows that $A_{d}$ is power bounded. By Lemma 4.1(a), the norm estimate (4.16) is equivalent to

$$
\left\|A_{d}^{n} A^{-1}\right\|=O\left(\frac{1}{n^{1 / 3}}\right) \quad n \rightarrow \infty .
$$

Define $\lambda_{k}:=1 / k-i k \in \sigma(-A)$ for $k \in \mathbb{N}$, and take $m \in \mathbb{N}$. Then

$$
\left\|A_{d}^{m n^{3}} A^{-3}\right\|=\sup _{k \in \mathbb{N}}\left|\frac{1-\lambda_{k}}{1+\lambda_{k}}\right|^{m n^{3}}\left|\lambda_{k}\right|^{-3} \geq\left|\frac{1-\lambda_{n}}{1+\lambda_{n}}\right|^{m n^{3}}\left|\lambda_{n}\right|^{-3} .
$$

for every $n \in \mathbb{N}$. We have that

$$
n^{3}\left|\frac{1-\lambda_{n}}{1+\lambda_{n}}\right|^{m n^{3}}\left|\lambda_{n}\right|^{-3}=\frac{n^{3}}{\left(\frac{1}{n^{2}}+n^{2}\right)^{\frac{3}{2}}}\left(1-\frac{4}{n^{3}+n+2+1 / n}\right)^{\frac{m n^{3}}{2}} .
$$


Since

$$
\left(1-\frac{4}{n^{3}+n+2+1 / n}\right)^{\frac{m n^{3}}{2}} \rightarrow e^{-2 m} \quad n \rightarrow \infty
$$

it follows that

$$
\liminf _{n \rightarrow \infty} n^{3}\left\|A_{d}^{m n^{3}} A^{-3}\right\| \geq e^{-2 m}
$$

By (4.18) with $m=1$, the norm estimate (4.16) is optimal in the sense that $\lim \sup _{n \rightarrow \infty} n\left\|A_{d}^{n} A^{-3}\right\|>0$. The norm estimate (4.17) and the substitution of $m=3$ into (4.18) imply that the optimal decay rate of $\left\|A_{d}^{n} A^{-1}\right\|$ is $1 / n^{1 / 3}$.

\section{Robustness analysis of polynomial stability}

As another application of the argument based on Lyapunov equations established in Sect. 3, we here extend to the case of polynomial stability the following result (Lemma 2.6 of [17]) on the preservation of uniform boundedness.

Lemma 5.1. Let $A$ be the generator of a uniformly bounded $C_{0}$-semigroup on a Hilbert space $H$. If $0 \in \varrho(A)$, then $A+r A^{-1}$ also generates a uniformly bounded $C_{0}$-semigroup semigroup on $H$ for every $r \geq 0$.

This robustness result can be extended to the case of strong stability and exponential stability; see p. 358 of [17].

It turns out that the class of perturbations $\left\{r A^{-1}: r>0\right\}$ results in at most only arbitrarily small loss of decay rates.

Proposition 5.2. Let $A$ be the generator of a uniformly bounded $C_{0}$-semigroup $(T(t))_{t \geq 0}$ on a Hilbert space $H$ such that $i \mathbb{R} \subset \varrho(A)$. If $\left\|T(t)(-A)^{-\alpha}\right\|=O(1 / t)$ as $t \rightarrow \infty$ for some $\alpha>0$, then the following assertions hold for every $r \geq 0$ :

(a) $A+r A^{-1}$ generates a uniformly bounded $C_{0}$-semigroup $\left(S_{r}(t)\right)_{t \geq 0}$ on $H$;

(b) $i \mathbb{R} \subset \varrho\left(A+r A^{-1}\right)$; and

(c) for every $\varepsilon \in(0,1)$,

$$
\left\|S_{r}(t)\left(-A-r A^{-1}\right)^{-\alpha}\right\|= \begin{cases}O(1 / t) & \text { if } \alpha>2 \\ O(\log t / t) & \text { if } \alpha=2 \\ O\left(1 / t^{1-\varepsilon}\right) & \text { if } 0<\alpha<2\end{cases}
$$

as $t \rightarrow \infty$.

We need three auxiliary results for the Proof of Proposition 5.2. First, we present a simple result on the resolvent set of $A+A^{-1}$.

Lemma 5.3. Let $A: D(A) \subset X \rightarrow X$ be a closed operator on a Banach space $X$. If $i \mathbb{R} \subset \varrho(A)$, then $i \mathbb{R} \subset \varrho\left(A+A^{-1}\right)$. 
Proof. Let $\omega \in \mathbb{R}$. A routine calculation shows that

$$
i \omega-A-A^{-1}=-\left(A-i \omega_{1} I\right)\left(A-i \omega_{2} I\right) A^{-1},
$$

where

$$
\omega_{1}:=\frac{\omega}{2}+\sqrt{1+\frac{\omega^{2}}{4}}, \quad \omega_{2}:=\frac{\omega}{2}-\sqrt{1+\frac{\omega^{2}}{4}} .
$$

From $i \mathbb{R} \subset \varrho(A)$, it follows that $A-i \omega_{1} I$ and $A-i \omega_{2} I$ are invertible in $\mathcal{L}(X)$. Hence,

$$
\left(i \omega-A-A^{-1}\right)^{-1}=-A\left(A-i \omega_{1} I\right)^{-1}\left(A-i \omega_{2} I\right)^{-1} \in \mathcal{L}(X) .
$$

Thus, $i \mathbb{R} \subset \varrho\left(A+A^{-1}\right)$.

Second, we present a result on the domain of fractional powers under bounded perturbations, which is used to prove (5.1) in the case $0<\alpha<2$.

Lemma 5.4. Let $X$ be a Banach space and $B \in \mathcal{L}(X)$. Suppose that $A$ and $A+$ $B$ generate $C_{0}$-semigroups on $X$ with negative growth bounds. Then the inclusion $D\left((-A)^{\alpha}\right) \subset D\left((-A-B)^{\beta}\right)$ holds for every $\alpha, \beta \in(0,1)$ with $\beta<\alpha$.

Proof. For $\alpha \in(0,1)$, define the abstract Hölder spaces of order $\alpha$ by

$$
\begin{aligned}
X_{\alpha} & :=\left\{x \in X: \lim _{\lambda \rightarrow \infty}\left\|\lambda^{\alpha} A R(\lambda, A) x\right\|=0\right\} \\
X_{\alpha}^{B} & :=\left\{x \in X: \lim _{\lambda \rightarrow \infty}\left\|\lambda^{\alpha}(A+B) R(\lambda, A+B) x\right\|=0\right\} .
\end{aligned}
$$

Since $A$ and $A+B$ generate $C_{0}$-semigroups with negative growth bounds, it follows from Proposition II.5.33 of [10] that

$$
X_{\alpha} \subset D\left((-A)^{\beta}\right) \subset X_{\beta}, \quad X_{\alpha}^{B} \subset D\left((-A-B)^{\beta}\right) \subset X_{\beta}^{B}
$$

for $0<\beta<\alpha<1$. It suffices to show that $X_{\alpha} \subset X_{\alpha}^{B}$ for every $\alpha \in(0,1)$. In fact, combining this inclusion with (5.2), we obtain

$$
D\left((-A)^{\alpha}\right) \subset X_{\alpha} \subset X_{\alpha}^{B} \subset D\left((-A-B)^{\beta}\right)
$$

for $0<\beta<\alpha<1$.

To obtain $X_{\alpha} \subset X_{\alpha}^{B}$, we use the identity

$$
R(\lambda, A+B)=R(\lambda, A)+R(\lambda, A) B R(\lambda, A+B) \quad \forall \lambda>0 .
$$

This yields

$$
\begin{aligned}
(A+B) R(\lambda, A+B)= & A R(\lambda, A)+B R(\lambda, A) \\
& +(A+B) R(\lambda, A) B R(\lambda, A+B)
\end{aligned}
$$


for every $\lambda>0$. There exists $M>0$ such that

$$
\|\lambda R(\lambda, A)\| \leq M, \quad\|\lambda R(\lambda, A+B)\| \leq M \quad \forall \lambda>0,
$$

and hence,

$$
\begin{aligned}
\|(A+B) R(\lambda, A)\| & \leq\|-I+\lambda R(\lambda, A)\|+\|B R(\lambda, A)\| \\
& \leq 1+M+\frac{M\|B\|}{\lambda} \quad \forall \lambda>0 .
\end{aligned}
$$

Consequently,

$$
\begin{aligned}
& \lim _{\lambda \rightarrow \infty}\left\|\lambda^{\alpha} B R(\lambda, A)\right\|=0 \\
& \lim _{\lambda \rightarrow \infty}\left\|\lambda^{\alpha}(A+B) R(\lambda, A) B R(\lambda, A+B)\right\|=0
\end{aligned}
$$

for every $\alpha \in(0,1)$. It follows from (5.3) that $X_{\alpha} \subset X_{\alpha}^{B}$.

Third, Lyapunov equations for $A$ are connected to those for $A+A^{-1}$ by the following result obtained in the proof of [17, Lemma 2.6].

Lemma 5.5. Let $A$ be the generator of a uniformly bounded $C_{0}$-semigroup on a Hilbert space $H$. Suppose that $0 \in \varrho(A)$, and take $\kappa>\left\|A^{-1}\right\|^{2}$. For every $\xi>0$, the self-adjoint solution $Q(\xi) \in \mathcal{L}(H)$ of the Lyapunov Eq. (3.14) satisfies

$$
\left(A+A^{-1}-\xi I\right)^{*} Q\left(\frac{\xi}{1+\kappa}\right)+Q\left(\frac{\xi}{1+\kappa}\right)\left(A+A^{-1}-\xi I\right) \leq-I \text { on } D(A) .
$$

We are now ready to prove Proposition 5.2.

Proof of Proposition 5.2. Since

$$
A+r A^{-1}=r^{1 / 2}\left(\left(r^{-1 / 2} A\right)+\left(r^{-1 / 2} A\right)^{-1}\right) \quad \forall r>0
$$

it suffices to prove the case $r=1$. By Lemma 5.1, $\left(S_{1}(t)\right)_{t \geq 0}$ is uniformly bounded. We see from Lemma 5.3 that $i \mathbb{R} \subset \varrho\left(A+A^{-1}\right)$.

It remains to prove the norm estimate (5.1) for $r=1$. For $\xi>0$, let $Q(\xi) \in \mathcal{L}(H)$ be the self-adjoint solution of the Lyapunov Eq. (3.14). Take $\kappa>\left\|A^{-1}\right\|^{2}$ and define

$$
Q_{1}(\xi):=Q\left(\frac{\xi}{1+\kappa}\right), \quad \xi>0 .
$$

By Lemma 5.5, $Q_{1}(\xi)$ satisfies

$$
\left(A+A^{-1}-\xi I\right)^{*} Q_{1}(\xi)+Q_{1}(\xi)\left(A+A^{-1}-\xi I\right) \leq-I \quad \text { on } D(A)
$$

for every $\xi>0$. Therefore,

$$
\frac{1}{2 \pi} \int_{-\infty}^{\infty}\left\|R\left(\xi+i \eta, A+A^{-1}\right) x\right\|^{2} d \eta \leq\left\langle x, Q_{1}(\xi) x\right\rangle
$$


for every $x \in H$ and every $\xi>0$; see Remark 2.3 of [17]. For $\gamma \in(0,1 / 2]$, define

$$
h_{\gamma}(\xi):= \begin{cases}\xi^{1-2 \gamma} & \text { if } 0<\gamma<1 / 2 \\ \frac{1}{\log (1 / \xi)} & \text { if } \gamma=1 / 2 .\end{cases}
$$

Then (a) and (b) of Lemma 3.3 show that

$$
\lim _{\xi \rightarrow 0+} h_{\gamma}(\xi)\left\langle x, Q_{1}(\xi) x\right\rangle=0
$$

for every $x \in D\left((-A)^{\alpha \gamma}\right)$.

First we consider the case $\alpha \geq 2$. Define $\gamma:=1 / \alpha$. Then $\gamma \in(0,1 / 2]$ and $\alpha \gamma=1$. Since $D\left((-A)^{\alpha \gamma}\right)=D\left(\left(-A-A^{-1}\right)^{\alpha \gamma}\right)$, it follows from (5.4) and (5.5) that

$$
\lim _{\xi \rightarrow 0+} h_{\gamma}(\xi) \int_{-\infty}^{\infty}\left\|R\left(\xi+i \eta, A+A^{-1}\right) x\right\|^{2} d \eta=0
$$

for every $x \in D\left(\left(-A-A^{-1}\right)^{\alpha \gamma}\right)$. Hence, (5.1) holds in the case $\alpha \geq 2$ by Proposition 3.1 (a) and (c).

Next we investigate the case $0<\alpha<2$. Choose $\gamma \in(0,1 / 2)$ and $\delta \in(0,1-\alpha \gamma)$ arbitrarily. For every $\alpha_{1}, \xi_{1}>0$,

$$
\begin{aligned}
D\left((-A)^{\alpha_{1}}\right) & =D\left(\left(-A+\xi_{1} I\right)^{\alpha_{1}}\right) \\
D\left(\left(-A-A^{-1}\right)^{\alpha_{1}}\right) & =D\left(\left(-A-A^{-1}+\xi_{1} I\right)^{\alpha_{1}}\right)
\end{aligned}
$$

by Proposition 3.1.9.a) of [18]. Since $A-\xi_{1} I$ and $A+A^{-1}-\xi_{1} I$ generate $C_{0^{-}}$ semigroups with negative growth bounds for every $\xi_{1}>0$, it follows from Lemma 5.4 that

$$
D\left(\left(-A-A^{-1}\right)^{\alpha \gamma+\delta}\right) \subset D\left((-A)^{\alpha \gamma}\right) .
$$

Therefore, (5.4) and (5.5) lead to

$$
\lim _{\xi \rightarrow 0+} \xi^{1-2 \gamma} \int_{-\infty}^{\infty}\left\|R\left(\xi+i \eta, A+A^{-1}\right) x\right\|^{2} d \eta=0
$$

for every $x \in D\left(\left(-A-A^{-1}\right)^{\alpha \gamma+\delta}\right)$. From Proposition 3.1(a), we have that

$$
\left\|T(t)\left(-A-A^{-1}\right)^{-\alpha-\delta / \gamma}\right\|=O\left(\frac{1}{t}\right) \quad t \rightarrow \infty .
$$

Since $\gamma \in(0,1 / 2)$ and $\delta \in(0,1-\alpha \gamma)$ were arbitrary, it follows from Lemma 2.3 that (5.1) holds in the case $0<\alpha<2$. 
Open Access. This article is licensed under a Creative Commons Attribution 4.0 International License, which permits use, sharing, adaptation, distribution and reproduction in any medium or format, as long as you give appropriate credit to the original author(s) and the source, provide a link to the Creative Commons licence, and indicate if changes were made. The images or other third party material in this article are included in the article's Creative Commons licence, unless indicated otherwise in a credit line to the material. If material is not included in the article's Creative Commons licence and your intended use is not permitted by statutory regulation or exceeds the permitted use, you will need to obtain permission directly from the copyright holder. To view a copy of this licence, visit http://creativecommons.org/licenses/ by/4.0/.

Publisher's Note Springer Nature remains neutral with regard to jurisdictional claims in published maps and institutional affiliations.

\section{REFERENCES}

[1] Arendt, W., Batty, C.J.K., Hieber, M., Neubrander, F.: Vector-valued Laplace Transforms and Cauchy Problems. Basel: Birkhäuser (2001)

[2] Bátkai, A., Engel, K.J., Prüss, J., Schnaubelt, R.: Polynomial stability of operator semigroups. Math. Nachr. 279, 1425-1440 (2006)

[3] Batty, C.J.K., Chill, R., Tomilov, Y.: Fine scales of decay of operator semigroups. J. Eur. Math. Soc 18, 853-929 (2016)

[4] Batty, C.J.K., Duyckaerts, T.: Non-uniform stability for bounded semi-groups on Banach spaces. J. Evol. Equ. 8, 765-780 (2008)

[5] Batty, C.J.K., Gomilko, A., Tomilov, Y.: A Besov algebra calculus for generators of operator semigroups and related norm-estimates. Math. Ann. 379, 23-93 (2021)

[6] Borichev, A., Tomilov, Y.: Optimal polynomial decay of functions and operator semigroups. Math. Ann. 347, 455-478 (2010)

[7] Cohen, G., Lin, M.: Remarks on rates of convergence of powers of contractions. J. Math. Anal. Appl. 436, 1196-1213 (2016)

[8] Curtain, R.F., Zwart, H.J.: An Introduction to Infinite-Dimensional Linear Systems Theory. New York: Springer (1995)

[9] Eisner, T.: Stability of Operators and Operator Semigroups. Basel: Birkhäuser (2010)

[10] Engel, K.J., Nagel, R.: One-Parameter Semigroups for Linear Evolution Equations. New York: Springer (2000)

[11] Gautschi, W.: Some elementary inequalities relating to the gamma and incomplete gamma function. J. Math. Phys. 38, 77-81 (1959)

[12] Gomilko, A.: Cayley transform of the generator of a uniformly bounded $C_{0}$-semigroup of operators. Ukrainian Math. J. 56, 1212-1226 (2004)

[13] Gomilko, A.: Inverses of semigroup generators: a survey and remarks. In: Études opératorielles, Banach Center Publ, pp. 107-142 (2017)

[14] Gomilko, A., Zwart, H.: The Cayley transform of the generator of a bounded $C_{0}$-semigroup. Semigroup Forum 74, 140-148 (2007)

[15] Gomilko, A., Zwart, H., Besseling, N.: Growth of semigroups in discrete and continuous time. Studia Math. 206, 273-292 (2011)

[16] Gomilko, A.M.: Conditions on the generator of a uniformly bounded $C_{0}$-semigroup. Funct. Anal. Appl. 33, 294-296 (1999)

[17] Guo, B.Z., Zwart, H.: On the relation between stability of continuous-and discrete-time evolution equations via the Cayley transform. Integr. equ. oper. theory 54, 349-383 (2006)

[18] Haase, M.: The Functional Calculus for Sectorial Operators. Basel: Birkhäuser (2006)

[19] Haase, M.: Lectures on Functional Calculus. 21st International Internet Seminar, Kiel Univ (2018). Retrieved May 27, 2021, from https://www.math.uni-kiel.de/isem21/en/course/phase1/ isem21-lectures-on-functional-calculus

[20] Liu, Z., Rao, B.: Characterization of polynomial decay rate for the solution of linear evolution equation. Angew. Math. Phys. 56, 630-644 (2005) 
[21] Nagy, B.S., Foiaş, C.: Harmonic Analysis of Operators on Hilbelt Space. North Holland Publishing Co. (1970)

[22] Ng, A.C.S., Seifert, D.: Optimal rates of decay in the Katznelson-Tzafriri theorem for operators on Hilbert spaces. J. Funct. Anal. 279, Art. no. 108799 (2020)

[23] Paunonen, L.: Perturbation of strongly and polynomially stable Riesz-spectral operators. Systems Control Lett. 60, 234-248 (2011)

[24] Paunonen, L.: Robustness of strongly and polynomially stable semigroups. J. Funct. Anal. 263, 2555-2583 (2012)

[25] Paunonen, L.: Robustness of polynomial stability with respect to unbounded perturbations. Systems Control Lett. 62, 331-337 (2013)

[26] Piskarev, S., Zwart, H.: Crank-Nicolson scheme for abstract linear systems. Numer. Funct. Anal. Optim. 28, 717-736 (2007)

[27] Rastogi, S., Srivastava, S.: Strong and polynomial stability for delay semigroups. J. Evol. Equ. 21, 441-472 (2021)

[28] Rozendaal, J., Seifert, D., Stahn, R.: Optimal rates of decay for operator semigroups on Hilbert spaces. Adv. Math. 346, 359-388 (2019)

[29] Seifert, D.: A quantified Tauberian theorem for sequences. Stud. Math. 227, 183-192 (2015)

[30] Seifert, D.: Rates of decay in the classical Katznelson-Tzafriri theorem. J. Anal. Math. 130, 329-354 (2016)

[31] Shi, D.H., Feng, D.X.: Characteristic conditions of the generation of $C_{0}$ semigroups in a Hilbert space. J. Math. Anal. Appl. 247, 356-376 (2000)

[32] Staffans, O.J.: Well-Posed Linear Systems. Cambridge, UK: Cambridge Univ. Press (2005)

[33] Tomilov, Y.: A resolvent approach to stability of operator semigroups. J. Operator Theory 46, 63-98 (2001)

[34] Weidmann, J.: Linear operators in Hilbert spaces. New York: Springer (1980)

\author{
Masashi Wakaiki \\ Graduate School of System Informatics \\ Kobe University \\ Nada, Kobe \\ Hyogo 657-8501 \\ Japan \\ E-mail:wakaiki@ruby.kobe-u.ac.jp
}

Accepted: 1 June 2021 